\title{
WHAT HAVE LEARNED ABOUT GAMMA RAY BURSTS FROM AFTERGLOWS?
}

\author{
Mario Vietri \\ Università di Roma III
}

\begin{abstract}
The discovery of GRBs' afterglows has allowed us to establish several facts: their distance and energy scales, the fact that they are due to explosions, that the explosions are relativistic, and that the afterglow emission mechanism is synchrotron radiation. On the other hand, recent data have shown that the fireball model is wrong when it comes to the emission mechanism of the true burst (which is unlikely to be synchrotron again) and that shocks are not external. Besides these relatively tame points, I will also discuss the less well established physics of the energy deposition mechanism, as well as the possible burst progenitors.
\end{abstract}

KEYWORDS: gamma rays: bursts - stars: neutron - black holes - relativity hydrodynamcis emission mechanisms

\section{INTRODUCTION}

Gamma ray bursts (GRBs) were discovered in 1969 (Klebesadel, Strong and Olson 1973) by American satellites of the Vela class aimed at verifying Russian compliance with the nuclear atmospheric test ban treaty. Though the discovery was made in 1969, the paper appeared only four years later because the authors had lingering doubts about the reality of the effects they had discovered. Since then, several thousands of bursts have been observed by a more than a dozen different satellites, but it is remarkable that the basic burst features outlined in the abstract of the 1969 paper (photons in the range $0.2-1.5 \mathrm{MeV}$, durations of $0.1-30 \mathrm{~s}$, fluences in the range $10^{-5}-2 \times 10^{-4}$ ergs $\mathrm{cm}^{-2}$ ) have remained substantially unchanged.

Current evidence (Fishman and Meegan 1995) has highlighted a wide (0.01 $100 \mathrm{~s}$ ) duration distribution, with hints of a bimodality which is claimed to correlate (at the $2.5 \sigma$ level) with spectral properties. All bursts' spectra observed so far are strictly non-thermal, and there has never been any confirmation by BATSE of a supposed thermal component (nor of cyclotron lines or precursors, for this matter) claimed in previous reports. A remarkable feature reported by BATSE is the bewildering diversity of light curves, ranging from impulsive ones (a spike followed by a slower decay, nicknamed FREDs for Fast Rise-Exponential Decay), to smooth ones, to long ones with amazingly sharp fluctuations, including even some with a strongly periodic appearance (two such examples are the 'hand' and the 'comb', so nicknamed from the number of high- $\mathrm{Q}$, regularly repeating sharp spikes). 


\section{BATSE Gamma-Ray Bursts}

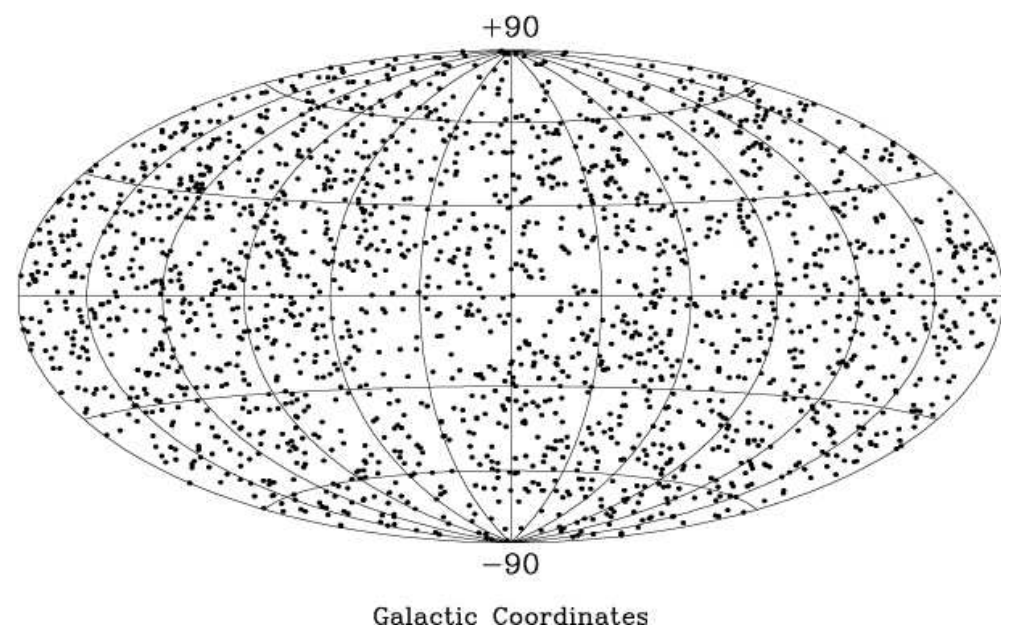

FIGURE 1. Burst distribution on the plane of the sky,

The most exceptional result from BATSE, though, was the sky distribution of the bursts (Fig.1). It was obvious from it that the bursts had to be extragalactic, as already discussed by theorists (Usov and Chibisov 1975, Paczyǹski 1986).

\section{AFTERGLOWS}

The next major step was triggered by BeppoSAX: in the summer of 1996, L. Piro and his coworkers located in archival data of the satellite the soft X-ray counterpart of a GRB (GRB 960720). They immediately conceived the idea of implementing a procedure to follow the next burst in real time, by re-orienting the whole satellite, after the initial detection by the Wide Field Cameras, so that the more sensitive Narrow Field Instruments could pinpoint the burst location to within 45 arcsecs, a feat never achieved in such short times, and by a single satellite. After an initial snafu (GRB 970111), the gigantic effort paid off with the discovery of the X-ray afterglow of GRB 970228 (Costa et al., 1997), immediately followed by the discovery of its fading optical counterpart (van Paradijs et al., 1997), obtained through a search inside the WFC error box, in perfect agreement with theoretical predictions (Vietri 1997a, Mèszàros and Rees 1997)円.

After the detection of the optical counterpart, the door was open to find the bursts' redshifts: Table I summarizes the status of our current knowledge (september

\footnotetext{
${ }^{1}$ There is an interesting lesson to be drawn from this: in case one should wonder why a soft $\mathrm{X}$ ray telescope was not placed onboard Compton to track GRBs, it was because of rivalries between different NASA subsections, the $X$-ray and the $\gamma$-ray divisions.
} 


\begin{tabular}{|c|c|c|}
\hline GRB & Z & $E_{\text {iso }}$ \\
\hline 970228 & 0.695 & $5 \times 10^{51} \mathrm{erg}$ \\
\hline 970508 & 0.835 & $2 \times 10^{51} \mathrm{erg}$ \\
\hline 971214 & 3.4 & $3 \times 10^{53} \mathrm{erg}$ \\
\hline 980703 & 0.93 & $3 \times 10^{53} \mathrm{erg}$ \\
\hline 990123 & 1.7 & $4 \times 10^{54} \mathrm{erg}$ \\
\hline 990510 & 1.6 & $2 \times 10^{53} \mathrm{erg}$ \\
\hline 990712 & 0.43 & \\
\hline
\end{tabular}

1999); bursts' luminosities are for isotropic sources. Two comments are in order. First, the bursts have prima facie a redshift distribution not unlike that of AGNs and of the Star Formation Rate (SFR). The initial hope that they might trace an even more distant and elusive Pop III, triggered by the fact that the second redshift detected was also the largest so far (GRB 971214, $z=3.4$ ), has now vanished. Second, in order to place the energy release of GRB 990123 in context, one should notice that $4 \times 10^{54}$ ergs is the energy obtained by converting the rest-mass of two solar masses, or, alternatively, the energy emitted by the whole Universe out to $z \approx 1$ within the burst duration. So, a single (perhaps double) star outshines the whole Universe.

Besides the distance and energy scales, the major impact of the discovery of afterglows has been the establishment of some key features of the fireball model (Rees and Mèszàros 1992):

1. bursts are due to explosions, as evidenced by their power-law behaviour;

2. the explosions are relativistic, as proved by the disappearence of radio flares;

3. the burst emission is due to synchrotron emission, as shown by the afterglow spectrum, and its optical polarization.

I will illustrate these points in the following, but, lest we become too proud, we should also remember that the fireball model has met some failures. The original version of the model (Mèszàros and Rees 1993) advocated the dissipation of the explosion energy at external shocks (i.e., those with the interstellar medium). Sari and Piran (1997), following a point originally made by Ruderman (1975) showed that these shocks smooth out millisecond timescale variability, which can only be maintained by the internal shocks proposed by Paczyǹski and Xu (1994). Also, the fireball model originally ascribed even the emission from the burst proper (as opposed to the afterglow) to optically thin synchrotron processes; I will discuss in the section Embarrassments why this is exceedingly unlikely. Furthermore, even the last tenet of mid-90s common wisdom, i.e., that bursts are due to neutron binary mergers, does not look too promising at the moment (since some bursts seem to 
be located inside star forming regions, incompatible with the long spiral-in time), though of course it is by no means ruled out yet.

\subsection{The fireball model}

Here, one may assume that an unknown agent deposits $10^{51}-10^{54}$ ergs inside a small volume of linear dimension $\approx 10^{6}-10^{7} \mathrm{~cm}$. The resulting typical energy density corresponds to a temperature of a few $M e V s$, so that electrons and positrons cannot be bound by any known gravitational field. In these conditions, optical depths for all known processes exceed $10^{10}$. The fluid expands because of its purely thermal pressure, converting internal into bulk kinetic energy. Parametrizing the baryon component mass as $M_{b} \equiv E / \eta c^{2}$, it can be shown that, for $1 \leq \eta \leq 3 \times 10^{5}$ (Mèszàros, Laguna, Rees 1993) the fluid achieves quickly (the fluid Lorenz factor increases as $\gamma \propto r)$ a coasting Lorenz factor of $\gamma \approx \eta$.

The requisite asymptotic Lorenz factor is dictated by observations: photons up to $\epsilon_{e x} \approx 18 \mathrm{GeV}$ have been observed by EGRET from bursts (Fishman and Meegan 1995). For these photons to evade collisions with other photons, and thus electron/positron pair production, it is necessary that, in the reference frame in which a typical burst photon (with $\epsilon \approx 1 \mathrm{MeV}$ ) and the exceptional photon are emitted, they appear as below pair production threshold: thus we must have $\epsilon^{\prime} \epsilon_{e x}^{\prime} \leq 2 m_{e} c^{2}$. Since $\epsilon^{\prime} \approx \epsilon / \gamma$, and similarly for the other photons, we find (Baring 1993)

$$
\gamma \approx 300\left(\frac{\epsilon}{1 M e V} \frac{\epsilon_{e x}}{10 G e V}\right)^{1 / 2} .
$$

From what we said above, we thus require a maximum baryon contamination, in an explosion of energy $E$, of $M_{b} \lesssim 10^{-6} M_{\odot}\left(E / 10^{51} \mathrm{erg}\right)(300 / \eta)$.

The energy release is now assumed to be in the form of an inhomogeneous wind, with parts having a Lorenz factor larger than parts emitted previously. This leads to shell collisions (the internal shock model) at radii $r_{s h}$ which allow a time-scale variablity $\delta t \approx r_{s h} / 2 \gamma^{2} c$; for $\delta t=1 \mathrm{~ms}, r_{s h} \approx 10^{13} \mathrm{~cm}$, which fixes the internal shock radii. Particle acceleration at these internal shocks and ensuing non-thermal emission is thought to lead to the formation of the burst proper. At larger radii, a shock with the surrounding ISM forms, and shell deceleration begins at a radius $r_{a g}=\left(3 E / 4 \pi n m_{p} c^{2} \gamma^{2}\right)^{1 / 3} \approx 10^{17} \mathrm{~cm}$ for a $n=1 \mathrm{~cm}^{-3}$ particle density typical of galactic disks. It is thought that the afterglow begins when the shell begins the slowdown, as this drives a marginally relativistic shock into the ejecta, thusly extracting a further fraction of their bulk kinetic energy.

\subsection{Why explosions}

The success of the fireball model lies in this, that it decouples the problem of the energy injection mechanism from the following evolution, which is, furthermore, an essentially hydrodynamical problem. It can be shown, in fact (Waxman 1997) that the evolution of the external shock is adiabatic, that the shock Lorenz factor 
decreases as $\gamma \propto r^{-3 / 2}$ because of the inertia of the swept-up matter, and thus $r$ scales with observer's time as $t=r / \gamma^{2} c \rightarrow \gamma \propto t^{-3 / 8}$ (for a radiative solution $\gamma \propto r^{-3 / 7}$, Vietri 1997b). If afterglow emission is due to optically thin synchrotron in a magnetic field in near-equipartition with post-shock energy density, it can be shown that $B \propto \gamma$, that the typical synchrotron frequency at the spectral peak $\nu_{m} \propto \gamma B \gamma_{e}^{2} \propto \gamma^{4}$ (where $\gamma_{e} \propto \gamma$ is the lowest post-shock electron Lorenz factor), and that $F\left(\nu_{m}\right) \propto t^{-3 \beta / 2}$, where $\beta$ is the afterglow spectral slope. As it can be seen, these expectations are based exclusively upon the hydrodynamical evolution (and the synchrotron spectrum), and are thus reasonably robust.

We thus expect power-law time decays, a characteristic of strong explosions (see the Sedov analogy!), with time- and spectral-indices closely related. This is what is observed everywhere, from the $\mathrm{X}$-ray through the optical to the radio, (see Piro and Fruchter, this volume), the few exceptions being discussed later on. In fact, the equality of the time-decay index of the $\mathrm{X}$-ray and optical data in afterglows of individual sources has been taken as the key element to show that emission in the different bands is due to the same source. Time indices in the $\mathrm{X}$-ray are in the range $0.7-2.2$ (Frontera et al., in preparation).

\subsection{Why synchrotron spectrum in the afterglow}

After having established that bursts are due to explosions, we happily learn that afterglows emit through synchrotron processes. In fig. 2 (Galama et al., 1998), we show the superposition of theoretical expectations for an optically thin synchrotron spectrum (including the cooling break at $\nu \approx 10^{14} \mathrm{~Hz}$ ) with observations for GRB 970508. The remarkable agreement is even more exciting as we remark that observations are not truly simultaneous, but are scaled back to the same time by means of the theoretically expected laws for time-decay, thus simultaneoulsy testing the correctness of our hydro. Another piece of evidence comes from the discovery of polarization in the optical afterglow of GRB 990510 (Fig. 3, Covino et al., 1999, Wijers et al., 1999). This polarization may appear small $(\approx 2 \%)$, but it is surely not due to Galactic effects: stars in the same field show a comparable degree of polarization, but along an axis different by about $50^{\circ}$. Also, polarization in the source galaxy is unlikely, because of a very stringent upper limit on the reddening due to this galaxy (Covino et al., 1999). The only remaining question mark is emission from an anisotropic source, but this would require a disk of $10^{18} \mathrm{~cm}$ to survive the intense $\gamma$ ray (and $\mathrm{X}$, and UV) flash: though not excluded, it does not look likely.

\subsection{Why relativistic expansion}

Radio observations of the first burst observed so far (GRB 970508, Frail et al., 1997) showed puzzling fluctuations by about a factor of 2 in the flux, over a time-scale of days, disappearing after about 30 days from the burst (Fig. 4). This extreme, and unique behaviour, was explained by Goodman (1997), who showed that it is due to interference of rays travelling along different paths through the ISM, and randomly deflected by the spatially varying refractive index of the turbulent ISM. 


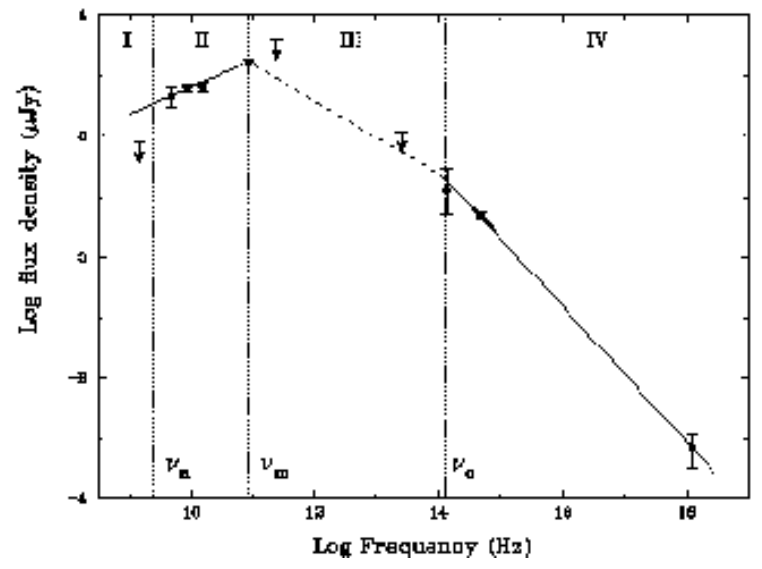

FIGURE 2. Simultaneous spectrum of the afterglow of GRB 970508, from Galama et al., 1998.

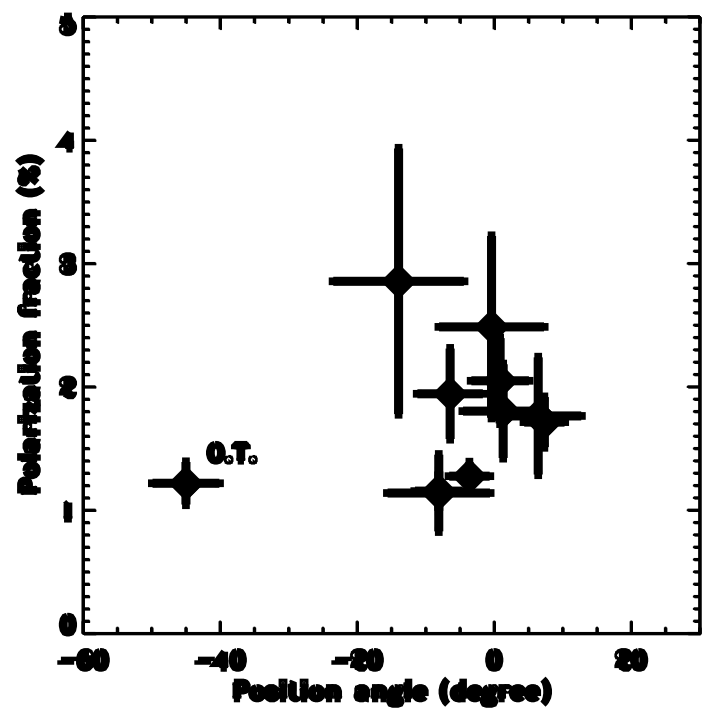

FIGURE 3. Polarization amplitude and position angle for optical afterglow of GRB 990510, from Covino et al., 1999 


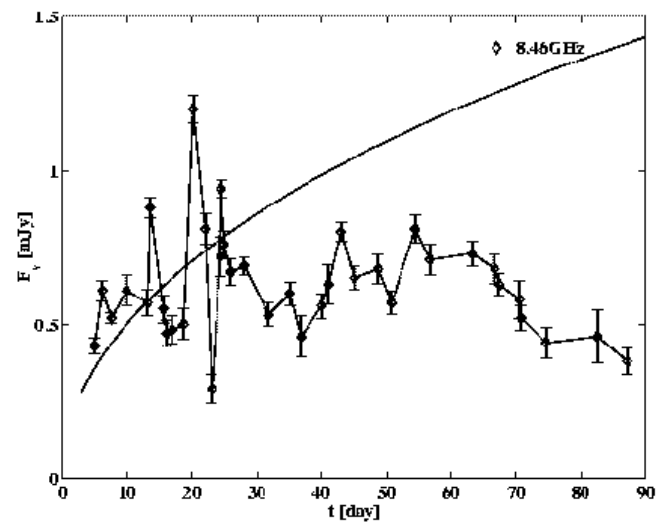

FIGURE 4. VLA observatons at $8.46 \mathrm{GHz}$ of the afterglow of GRB 970508, from Waxman,Frail and Kulkarni 1998.

The wonderful upshot of this otherwise marginal phenomenon, is that these effects cease whenever the source expands beyond a radius

$$
R=10^{17} c m \frac{\nu_{10}^{6 / 5}}{d_{s c, k p c} h_{75}}\left(\frac{S M}{10^{-2.5} m^{-20 / 3} k p c}\right)^{-3 / 5},
$$

where $\nu_{10}$ is the radio observing frequency in units of $10^{10} \mathrm{~Hz}, d_{s c, k p c}$ is the distance of the ISM from us (assumed to be a uniform scattering screen), and $S M$ is the Galactic scattering measure, scaled to a typical Galactic value. The existence of interference effects is made more convincing by the amplitude of the average increase (a factor of 2 , as observed), the correctness in the prediction of the timeinterval between different peaks, and of the decorrelation bandwidth. Since flares disappear after about 30 days, it means that the average speed of the radio source is $R / 30$ days $=3 \times 10^{10} \mathrm{~cm} \mathrm{~s}^{-1}$. So we see directly that GRB 970508 expanded at an average speed of $c$ over a whole month, giving us a direct observational proof that the source is highly relativistic. This proof is completely equivalent to superluminal motions in blazars, and is the strongest evidence in favor of the fireball model.

\subsection{GRB 970508: our best case}

The afterglow of GRB 970508 is our best case so far: it is in fact a burst for which not only do we know the redshift, but also a radio source that has been monitored for more than 400 days after the explosion (Frail, Waxman and Kulkarni 2000). Through these observations we can see the transition to a sub-relativistic regime at $t \approx 100 d$, measure the total energetics of the following Sedov phase (unencumbered 
by relativistic effects!) $E_{N e w}=5 \times 10^{50} \mathrm{ergs}$, determine two elusive parameters, $\epsilon_{e q}=0.5$ and $\epsilon_{B}=0.5$ (the efficiencies with which energy is transfered to postshock electrons by protons, and with which an equipartition field is built up), and the density of the surrounding medium $n \approx 0.4 \mathrm{~cm}^{-3}$. All of these values look reasonable (perhaps $\epsilon_{e q}$ and $\epsilon_{B}$ exceed our expectations by a factor of 10, a fact that could be remedied by introducing a slight density gradient which would keep the shock more efficient), so that our confidence in the external-shock-in-the-ISM model is boosted.

Another precious consequence of these late-time observations is that they yield information on beaming and energetics. In fact, GRB 970508 appeared to have a kinetic energy of $E_{r e l}=5 \times 10^{51} \mathrm{erg}$ when in the relativistic phase, a measurement which can be reconciled with $E_{N e w}$ (remember that the expansion is adiabatic, so that we must have $E_{N e w}=E_{r e l}$ !) only if the unknown beaming angle, assumed $=4 \pi$ in deriving $E_{r e l}$, is smaller than $4 \pi$ by the factor $E_{N e w} / E_{r e l}$; we thus have the only measurement of $\delta \Omega / 4 \pi=0.1$, so far. This already rules out all classes of models requiring unplausibly large amounts of beaming, $10^{-8}$ or even beyond. Hopefully, more such measurements will come in the future, since this observationally heavy method is subject to many fewer uncertainties than the competing method of trying to locate breaks in the time-decay of afterglows. Also, the radiative efficiency of the burst can be estimated: correcting the observed burst energy release $E_{G R B}=2 \times 10^{51} \mathrm{erg}$ for the same beaming factor, the radiative efficiency is $E_{G R B} \delta \Omega / 4 \pi /\left(E_{N e w}+E_{\text {rel }} \delta \Omega / 4 \pi\right)=0.3$, again a unique determination. Notice however that this figure is subject to a systematic uncertainty: we do not know whether the beaming fraction is the same for the burst proper and for the afterglow.

\section{EMBARRASSMENTS}

Something is rotten in the fireball kingdom as well, namely, departures from pure power-law behaviours, and the spectra of the bursts proper.

\subsection{Unpowerlawness}

Departures from power-laws are expected when one considers the extremely idealized character of the solutions discussed so far: perfect spherical symmetry, uniform surrounding medium, smooth wind from the explosion, $\epsilon_{e q}$ and $\epsilon_{B}$ constant in space and time. The tricky point here is to disentangle these distinct factors. In GRB 970508 and GRB 970828 (Piro et al., 1999, Yoshida et al., 1999) a major departure was observed in the $\mathrm{X}$-ray emission, within a couple of days from the burst; they constitute the single, largest violations observed so far, in terms of number of photons. It is remarkable that spectral variations were simultaneously observed, and that both bursts showed traces (at the $2.7 \sigma$ significance level) of an iron emission line. The similarity of the bursts' behaviour argues in favor of the reality of these spectral features, which have been interpreted as thermal emission from a surrounding stellar-size leftover, pre-expelled by the burst's progenitor (Lazzati et al., 1999, 
Vietri et al., 1999). Clearly, these departures hold major pieces of information on the bursts' surroundings, and the nature of bursts' progenitors.

It has been argued (Rhoads 1997) that, whenever the afterglow shell decelerates to below $\gamma \approx 1 / \theta$, where $\theta$ is the beam semi-opening angle, emission should decrease because of the lack of emitting surface, compared to an isotropic source. But, in view of the existence of clear environmental effects (GRB 970508 and GRB 970828), it appears premature to put much stock in the interpretation of time-power-law breaks as due to beaming effects. And equally, it appears to this reviewer that the same comment applies to the interpretation of a resurgence of flux as due to the appearance of a SN remnant behind the shell. The major uncertainty here is the non-univoqueness of the interpretation: Waxman and Draine (2000) have shown that effects due to dust can mimic the same phenomenon.

\subsection{Bursts' spectra}

A clear prediction of the emission of optically thin synchrotron is that the lowphoton-energy spectra should scale like $d N_{\nu} / d \nu \propto \nu^{\alpha}$, with $\alpha=-3 / 2$, since the emission is in the fast cooling regime. Within thin synchrotron, there is no way to obtain $\alpha>-3 / 2$. This early-recognized requirement (Katz 1994) is so inescapable that it has been dubbed the 'line of death'. Observations are notoriously discordant with this prediction. Preece et al.(1999) have shown that, for more than 1000 bursts, $\alpha$ is distributed like a bell between -2 and 0 , with mean $\bar{\alpha} \approx-1$. The tail of this distribution also contains a few tens of objects with $\alpha \approx+1$. An example of these can be found in Frontera et al., 1999 (GRB 970111), which is instructive since BeppoSAX has better coverage of the critical, low-photon-energy region. In particular, BATSE seems to loose sensitivity below $\approx 30 \mathrm{keV}$, but this is still not enough to explain away the discrepancy with the theory. Also, Preece et al., 1999, showed that the time-integrated spectral energy distribution has a peak at a photon energy $\epsilon_{p k} \approx 200 \mathrm{keV}$, and that $\epsilon_{p k}$ has a very small variance from burst to burst. Again, this does not seem dependent upon BATSE's lack of sensistivity above $700 \mathrm{keV}$, and again this has no explanation within the classic fireball model.

Any theorist who worked on blazars will say that the root of the disagreement is the neglect of Inverse Compton processes, but the trick here is not to identify the culprit, on which everyone agrees, but to devise a fireball model that smoothly incorporates it. One should remember that the details of the fireball evolution are generic, i.e., they do not depend upon any detailed property of the source, so that things like the radius at which the fireball becomes optically thin (to pairs or baryonic electrons), the radius at which acceleration ends, the equipartition magnetic field, and so on, are all reliably and inescapably fixed by the outflow's global properties. A step toward the solution has been made by Ghisellini and Celotti (1999) who remarked that at least some bursts have compactness parameters $l=10\left(L / 10^{53} \mathrm{erg} \mathrm{s}^{-1}\right)(300 / \gamma)^{5} \gg 1$. Under these conditions, a pair plasma will form, nearly thermalized at $k T \approx m_{e} c^{2}$, and with Thomson optical depth $\tau_{T} \approx 10$. The modifications which this plasma will bring to the burst's spectrum are currently 
unknown, but it may be remarked that this configuration will be optically thick to both high-energy synchrotron photons due to non-thermal electrons accelerated at the internal shocks, and to low-energy cyclotron photons emitted by the thermal plasma, but it will be optically thin in the intermediate region reached by cyclotron photons upscattered via IC processes off non-thermal electrons. A model along this line (i.e., upscattering of cyclotron photons by highly relativistic electrons) is in preparation (Vietri 2000a), but it remains to be seen whether it (like any other model, of course) can simultaneously explain the spectral shape and the narrow range of the spectral distribution peak energy $\epsilon_{p k}$.

\section{ON THE CENTRAL ENGINE}

As remarked several times already, the fireball evolution is independent of the source nature. The only exisiting constraint is the maximum amount of baryon contamination, which is

$$
M_{b}=\frac{E}{\eta c^{2}}=10^{-6} M_{\odot} \frac{E}{10^{51} \mathrm{erg}} \frac{300}{\gamma} .
$$

This is a remarkably small value: since the inferred luminosities exceed the Eddington luminosity by 13 orders of magnitude, they clearly have all it takes to disrupt a whole star, no matter how compact. Yet, the energy deposition must somehow occur outside the main mass, lest the explosion be slowed down to less relativistic, or even possibly Newtonian speeds. In order to satisfy this constraint, it has emerged that the most favorable configuration has a stellar-mass black hole $\left(M_{B H} \approx 3-10 M_{\odot}\right)$ surrounded by a thick torus of matter $\left(M_{t} \approx 0.01-1 M_{\odot}\right.$, with $\left.\rho \approx 10^{10} \mathrm{~g} \mathrm{~cm}^{-3}\right)$. The presence of a black hole is not required by observations in any way: models involving neutron stars are still admissible, the advantage of having a black hole being only the deeper potential well: you get more energy out per unit accreted mass. The configuration thusly envisaged has a cone surrounding the symmetry axis devoid of baryons, since all models leading to this configuration have large amounts of specific angular momentum, and thus baryons close to the rotation axis either are not there, or have accreted onto the black hole due to their lack of centrifugal support.

\subsection{Energy release mechanism}

There are two major mechanisms for energy release discussed in the literature, the first one to be proposed (Berezinsky and Prilutskii 1986) being the reaction $\nu+\bar{\nu} \rightarrow$ $e^{-}+e^{+}$. Neutrinos have non-negligible mean free paths in the tori envisaged here, so that this annihilation reaction will take place not inside tori themselves, where they are preferentially generated because densities are highest, but in a larger volume surrounding the source. This is both a blessing and a disgrace: by occupying a larger volume, the probability that every neutrino finds its antiparticle to annihilate decreases, but then the energy is released in baryon-cleaner environments. The problem, though complex, is eminently suitable for numerical simulations, showing 
(Janka et al., 1999, and references therein) that about $10^{50}$ ergs can be released this way, above the poles of a black hole where less than $10^{-5} M_{\odot}$ are found.

Highly energetic bursts cannot be reproduced by this mechanism, due to its low efficiency: the second mechanism proposed involves the conversion of Poynting flux into a magnetized wind. The basic physical mechanisms are well-known (Usov 1992) since they have been studied in the context of pulsar emission: electrons are accelerated by a motional electric field $\vec{E}=\vec{v} \wedge \vec{B} / c$ due to the rotation of a sufficiently strong magnetic dipole, attached either to a black hole, or to the torus. Photons are then produced by synchrotron or curvature radiation, and photon/photon collisions produce pairs, to close the circle and allow looping. In order to carry away $10^{51} \mathrm{erg} \mathrm{s}^{-1}$, a magnetic field of $\approx 10^{15} \mathrm{G}$ is required. This is not excessive, since it is about three orders of magnitude below equipartition with torus matter, and because such fields already exist in nature, see SGR 1806-20 and SGR 1900+140: the key point is to understand whether some kind of dynamo effect can lead to these high values within the short allotted time.

Depending upon whether the open magnetic field lines extending to infinity are connected to the black hole or to the torus, the source of the energy of the outflow will be the rotational energy of the black hole (the so-called Blandford-Znajek effect) or of the torus. The first case is traditionally discussed in the context of AGNs (Rees, Blandford, Begelman and Phinney 1984), but it is harshly disputed whether the energy outflow may be actually dominated by the black hole rather than by the disk (Ghosh and Abramowicz, 1997, Livio, Ogilvie and Pringle 1998). On the other hand, the torus looks ideal as the source of a dynamo: its large shear rate, the presence of the Balbus-Hawley instability to convert polidal into toroidal flux, and the possible presence of the anti-floating mechanism inhibiting ballooning of the magnetic field (Kluzniak and Ruderman 1998), all seem to favor the existence of a fast dynamo. It should also be remarked that the configuration of the magnetic field in this problem is known: in fact, the configuration discussed in Thorne et al., 1986 for black holes, only uses the assumptions of steady-state and axial symmetry, and is thus immediately extended to magnetic fields anchored to the torus. What is really required here is a first order study, of the sort published by Tout and Pringle (1992) on angular momentum removal from young, pre-main-sequence stars via magnetic stresses, and on the associated $\alpha-\omega$ dynamo. Until such studies are made, it will be premature to claim that neutrino annihilations are responsible for the powering of GRBs.

\subsection{Progenitors}

There is no lack of proposed progenitors, but I will discuss only binary neutron mergers (Narayan, Paczyǹski and Piran 1992), collapsars (Woosley 1993, Paczyǹski 1998) and SupraNovae (Vietri and Stella 1998, 1999).

Clearly, NS/NS mergers is the best model on paper: it involves objects which have been detected already, orbital decay induced by gravitational wave emission is shown by observations to work as per the theory, and numerical simulations by 
Janka's group show that a neutrino-powered outflow in baryon-poor matter can be initiated. The major theoretical uncertainties here concern bursts' durations and energetics: all numerical models produce short bursts $(\approx 0.1 s)$ with modest energetics, $E<10^{51} \mathrm{erg}$. This is a direct consequence of the mechanism for powering the burst: large, super-Eddington luminosities are carried away by neutrinos, leading to a large mass influx, but only a small fraction, $1-3 \%$, can be harnessed for the production of the burst. Furthermore, we cannot invoke large beaming factors in this case: the outflow is only marginally collimated, in agreement with expectations that an accretion disk with inner and outer radii $R_{\text {out }} / R_{\text {in }} \approx$ a few (for the case at hand) can only produce a beam semi-opening angle of $R_{\text {in }} / R_{\text {out }}$. So, perhaps, this model may account for the short bursts, but it should be remembered that nothing of what was discussed above pertains to this subclass: BeppoSAX (and thus all BeppoSAX-triggered observations) can only detect long bursts.

On the other hand, future space missions, whether or not able to locate short bursts, can provide a decisive test of this model, provided they can follow with sufficient sensitivity a given burst for several hours. This model, in fact, is the only one proposed so far according to which some explosions should take place outside galaxies: according to Bloom, Sigurdsson and Pols (1999), about 50\% of all bursts will be located more than $8 \mathrm{kpc}$ from a galaxy, and $15 \%$ in the IGM. This characteristic is testable without recourse to optical observations. In fact, the afterglow begins with a delay (as seen by an outside observer) of $t_{d}=\left(r_{a g}-\right.$ $\left.r_{s h}\right) / \gamma^{2} c \approx r_{a g} / \gamma^{2} c$, which varies greatly depending upon the environment in which the burst takes place:

$$
t_{d}= \begin{cases}15 \mathrm{~s} & \text { ISM, } \mathrm{n}=1 \mathrm{~cm}^{-3} \\ 5 \mathrm{~min} & \text { galactic halo, } \mathrm{n}=10^{-4} \mathrm{~cm}^{-3} \\ 4 \mathrm{~h} & \text { IGM, } \mathrm{n}=10^{-8} \mathrm{~cm}^{-3}\end{cases}
$$

Between the burst proper and the beginning of the power-law-like afterglow, thus a silence of recognizable duration is expected (Vietri 2000b).

Collapsars are currently in great vogue as a possible source of GRBs: the large amoung of energy available as the core of a supermassive star collapses directly to a black hole is in fact very attractive, even though (again!) the limited efficiency of the reaction $\nu+\bar{\nu} \rightarrow e^{-}+e^{+}$makes most of this energy unavailable. Here too there is some evidence that these objects must exist (Paczyǹski 1998), and numerical simulations again showing energy preferentially deposited along the hole rotation axis are also available (McFayden and Woosley 1999). Here however, what is truly puzzling is how the outflow can pierce the star's outer layers without loading itself with baryons: we should remember that at most $10^{-6} M_{\odot}$ can be added to $10^{51} \mathrm{erg}$ : more baryons imply a proportionately slower outflow. The argument is that the dynamical timescale of the outer layers of a massive stars is of order of a few hours, so that, even if the core collapses and pressure support is removed, nothing will happen during the energy release phase: the outflow must pierce its way through. Two processes seem especially dangerous: Rayleigh-Taylor instability of the fluid heated-up by neutrino annihilations as it is weighed upon by the colder, denser 
outer layers, and Kelvin-Helmholtz instability after the hot fluid has pierced the outer layers and is passing through the hole. It is well-known that the non-linear development of these instabilities leads to mass entrainment, and that the time-scale for the development of these instabilities is very fast. Furthermore, the baryonfree outflow may be 'poisoned' by baryons to a deadly extent, even if numerical simulations, with their finite resolution, were to detect nothing of the kind.

The third class of models, SupraNovae, concerns supramassive neutron stars which are stabilized against self-gravity by fast rotation, to such an extent that they cannot be spun down to $\omega=0$ because they implode to a black hole. As the star's residual magnetic dipole sheds angular momentum, this is exactly the fate to be expected for the whole star, except for a small equatorial belt, whose later accretion will power the burst. It is easy to show that this implosion must take place in a very baryon-clean environment. The major uncertainties here concern the channels of formation and the existence of this equatorial belt. Two channels of formation have been proposed: direct collapse to a supramassive configuration (Vietri and Stella 1998) and slow mass accretion in a low-mass X-ray binary (Vietri and Stella 1999). Both are possible, though none yet is supported by observations. The existence of the left-over belt has recently been questioned by Shibata, Baumgarte and Shapiro (1999), who however simulated the collapse of neutron stars with intermediate equations of state, which are entirely (or nearly exactly so) contained inside the marginally stable orbit even before collapse: clearly, these must be swallowed whole by the resulting black hole. Soft equations of state are free of this objection, and are thus much more likely to leave behind an equatorial belt. The soft EoSs are especially favored since the neutron stars must survive the $r$-mode instability, and thus soft EoSs (Weber 1999) would be in any case required. So one might say that the existence of these stars hinges on one uncertainty only, the EoS of nuclear matter. Besides the baryon-clean environments, SupraNovae have another advantage over rival modlels: only the lowest density regions would be left behind, precisely those with the smallest neutrino losses. The powering of the burst can thus occur through accretion caused by removal of angular momentum by magnetic stresses, without the parallel, unproductive, neutrino generation.

\section{CONCLUSIONS}

It is difficult to end on an upbeat note: we cannot expect in the near future a rate of progress similar to the one we witnessed in the past three years. In particular, it may be expected that the next flurry of excitement will come with the beginning of the SWIFT mission, which promises to collect relevant data (redshifts, galaxy types, location within or without galaxies, absorption or emission features in the optical and in the $\mathrm{X}$-ray) for a few hundred bursts. This data will nail the major characteristics of the environment (at large) in which bursts take place, and we may be able to rule out a few models. On the other hand, the energy release process, shrouded as it is in optical depths $>10^{10}$, will remain mysterious, our only hope in this direction being gravitational waves. 
Judging by the analogy with radio pulsars, this will correspond to the flattening of the learning curve. Aside from this, we may hope to locate the equivalent of the binary radio pulsar, but, differently from Jo Taylor, we have to be awfully quick in grabbing it.

\section{ACKNOWLEDGEMENTS}

Thanks are due to Gabriele Ghisellini, who wisely steered me away from synchrotron emission, and toward the true light of Inverse Compton.

\section{REFERENCES}

??aring, M., 1993, Ap.J., 418, 391. ??erezinsky, V.S.,, Prilutskii, O.F., 1986, Astr.Ap., 175, 309. ??loom, J.S., Sigurdsson, S., Pols, O.R., 1999, MNRAS, 305, 763. ??osta, E., et al., 1997, Nature, 387, 783. ??ovino, S., et al., 1999, Astr.Ap., 348, L1. ??ishman, G.J., Meegan, C.A., 1995, ARAA, 33, 415. ??rail, D., et al., 1997, Nature, 389, 261. ??rail, D., Waxman, E., Kulkarni, S., 2000, Ap.J., submitted, astro-ph 9910319. ??rontera, F., et al., 1999, Ap.J.S., in press, astro-ph 9911228. ??alama, T., et al., 1998, Ap.J.L., 500, L97. ??hisellini, G., celotti, A., 1998, Ap.J.L., 511, L93. ??hosh, P., Abramowicz, M.A., 1997, MNRAS, 292, 887. ??oodman, J.J., 1997, New Astr., 2, 449. ??anka, T., Eberl, T., Ruffert, M., Fryer, C.L., 1999, Ap.J.L., 527, L39. ??atz, J., 1994, Ap.J., 422, 248. ??lebesadel, R.W., Strong, I.B., Olson, R.A., 1973, Ap.J.L., 182, L85. , ??luzniak, W., Ruderman, M., 1998, Ap.J.L., 505, L113. ??ivio, M., Ogilvie, G.I., Pringle, J.E., 1999, Ap.J., 512, 100. ??azzati, D., Campana, S., Ghisellini, G., MNRAS, 1999, 304, L31. ??c Fayden, A., Woosley, S.E., 1999, Ap.J., 524, 262. ??észáros, P. Laguna, P., Rees, M.J., 1993, Ap.J., 415, 181. ??észáros, Rees, M.J., 1993, Ap.J., 405, 278. ??észáros, Rees, M.J., 1997, Ap.J., 476, 232. ??arayan, R., Paczynski, B., Piran, T., 1992, Ap.J.L., 395, L83. ??aczyǹski, B., 1986, Ap.J.L., 308, L43. ??aczyǹski, B., 1998, Ap.J.L., 494, L45. ??aczyǹski, B., Xu, G., 1994, Ap.J., 427, 708. ??iro, L., et al., 1999, Ap.J.L., 514, L73. ??reece, R.D., et al., 1999, Ap.J.S., in press, astro-ph. 9908119. ??ees, M.J., Begelman, M.C., Blandford, R.D., Phinney E.S., 1984, Nature, 295, 17. ??ees, M.J., Mèszàros, P., 1992, MNRAS, 258, P41. ??hoads, J., 1997, Ap.J.L., 487, L1. ??uderman, R., 1975, Ann. NY. Acad. Sci., 262, 164. ??ari, R., Piran, T., 1997, Ap.J., 485, 270. ??hibata, M., Baumgarte, T.W., Shapiro, S.L., 1999, Phys.Rev.D, submitted, astro-ph. 9911308. ??horne, K.S., Price, R.H., MacDonald, D.A., 1986, Black holes: the membrane paradigm, Yale:New Haven, Yale Univ. Press. ??out, C.A., Pringle, J.E., 1992, MNRAS, 256, 269. ??sov, V.V., 1992, Nature, 357, 472. ??sov, V.V., Chibisov, G.V. Soviet Astr., 19, 115. ??an Paradijs, J., et al., 1997, Nature, 386, 686. ??ietri, M., 1997a, Ap.J.L., 478, L9. ??ietri, M., 1997b, Ap.J.L., 488, L105. ??ietri, M., 2000a, in preparation. ??ietri, M., 2000b, Ap.J.L., submitted. ??ietri, M., Perola, G.C., Piro, L., Stella, L., 1999, MNRAS, 308, P29. ??ietri, M., Stella, L., 1998, Ap.J.L., 507, L45. ??ietri, M., Stella, L., 1999, Ap.J.L., 527, L43. ??axman, E., 1997, Ap.J.L., 489, L33. ??axman, E., Draine, B.T., 2000, Ap.J., in press, astro-ph. 9909020. ??axman, E., Frail, D., Kulkarni, D., 1998, Ap.J., 497, 288. ??eber, F., 1999, in Pulsars as astrophysical laboratories for nuclear and particle physics, Bristol, U.K.; Institute of Physics. ??ijers, R.A.M.J., et al., 1999, Ap.J.L., 523, L33. ??oosley, S., 1993, Ap.J., 405, 273. ??oshida, A., et al., 1999, Astr.Ap.S., 138, 433. 